\title{
A Randomized Controlled Trial (Ret) on the Effects of Frequency Of Application Of Spinal Stabilisation Exercises On Plasma Serotonin Levels In Participants With Chronic Low Back Pain
}

\author{
*Sokunbi Ganiyu Oluwaleke (PhD), **Watt Peter $(\mathrm{PhD}), * * *$ Moore Ann \\ $(\mathrm{PhD})$ \\ *Department of Medical Rehabilitation, College of Medical Sciences, University of Maiduguri, Nigeria \\ **Chelsea School of Sport University of Brighton, UK \\ ***Clinical Research Center of University of Brighton, UK
}

\begin{abstract}
Objectives: The purpose of this study is to investigate the effects of frequency of application of spinal stabilization exercises on plasma levels of serotonin in participants with chronic low back disorders.

Methods: Eighty four participants (34 males, 50 females) volunteered to take part in this study. Plasma level of serotonin was measured with Enzyme linked Immuno absorbent assasy (ELISA) technique. Participants were randomly assigned to one of the exercise groups where they carried out exercise training at different frequencies i.e. once weekly (1wkly), twice weekly (2wkly)and three times a week (3wkly) or the control (no exercise group). Data were collected at baseline, after six weeks of exercise treatment and at 18 week follow up after exercise treatment.

Results: The result showed that plasma levels of serotonin increases significantly in all the exercises groups after 6 weeks of treatment with the highest increase in the three times a week $(P<0.05)$. Surprisingly, only the three times a week (3wkl) showed significant increase in plasma serotonin level at three months follow up $(P<0.05)$ which was not present in all other exercise groups.

Conclusion: The result of this study suggested that stabilization exercises carried out three times a week could be associated perhaps be associated with an optimal biochemical and long term therapeutic effects of spinal stabilization exercise via increase in production of plasma serotonin levels.
\end{abstract}

Key words: Chronic back pain, stabilization exercises, serotonin, $R C T$

\section{Introduction}

Back pain is one of the most common complaints in medical practice. But despite this, the efficacies of many treatments remain unproven and specialty biased often dominates the choice of preventive and therapeutic intervention ${ }^{1}$.

A recent focus in the physiotherapy management of chronic low back pain has been to identify specific muscles that are best able to stabilize the back and to carry out spinal stabilization exercises to enhance those muscles whose primary function is considered dynamic stabilization and segmental control of the spine ${ }^{2}$. Currently there is evidence demonstrating the efficacy of stabilization exercises on pain, functional disability and quality of life in sufferers with acute and chronic low back disorders ${ }^{2}$.The effects of stabilization exercise approach may be multifactorial involving physiological, psychological and other mechanisms yet to be well investigated ${ }^{3}$.

The likelihood of muscular contraction during spinal stabilization exercises providing sensory input to activate different pain inhibitory mechanisms appeared to have been suggested to little investigation. It is generally accepted that serotonin is involved in the mechanism of exercises induced analgesia ${ }^{4}$. Recent preliminary studies suggested an increase in the plasma level of serotonin produced with the use of stabilization exercises as one of the main facilitation techniques behind the efficacy of stabilization exercises ${ }^{3,5}$.

However the question of what is an appropriate dosage of treatment that can be expected to achieve a desired outcome in terms of plasma serotonin levels were not addressed in Sokunbi et al's studies ${ }^{3,5}$. Also, there is dearth need for a large randomized controlled trials with more participants with low back pain in order to make the study more generalizable to population of patients that suffer with low back pain. Thus, the purpose of this study is to investigate, in a randomized controlled trials, the effects of frequency of application of spinal stabilization exercises on plasma levels of serotonin in participants with chronic low back disorders

\section{Methods}

Ethical approval for this study was obtained from the Research Ethics Committee of the University of Brighton, UK. 


\section{Participants}

Eighty four participants, 50 females, 34 males with chronic low back pain volunteered to participate in this study. A written informed consent was obtained from all participants. Prior to this participants information sheet was provided for the participants so that they could understood what the study would involve. They were given the option to take part and are able to drop out of this study at any time if they choose to

\section{Inclusion Criteria}

Only those participants who met the inclusion criteria were allowed to participate in the study i.e. those with mechanical non specific low back pain of at least three months duration with and without pain radiating to one or both lower limbs. The acceptable age range was set at 18 to 60 years.

\section{Exclusion Criteria}

Participants were excluded from the study if they had a history of medical or surgical conditions which might hinder exercise performance, recent fracture of the spine (over the past 12 months), history of operative intervention for the lower back problems, pregnant women and participants with metastatic disease and evidence of serious spinal pathology (red flags). Other exclusion criteria include history of neurosis, depressive symptomatology and other low back pain yellow flag factors. All these exclusion criteria could potentially skew the data, thus any participants who demonstrate one or more of these criteria were excluded.

\section{Randomization of participants into study groups}

Microsoft excel 2000 were used to generate a list of randomized numbers which was used to randomly assigned subjects into the control and the three different exercise groups i.e. once weekly, twice weekly and three times a week.

\section{Sample size estimation}

Sample size estimation for the present RCT was based on the results of our preliminary study ${ }^{5}$ whereby participants showed at least $18 \%$ increase in plasma serotonin level in response to a similar exercises. To yield a power of $80 \%$ with a significant level of 0.05 , a sample size of at least 15 subjects in each group was required (sample size estimation was determined by power analysis sample size software).

\section{Pre intervention assessments}

Assessment of Demographic variables

Participants' heights, Weight were measured. Information regarding age, mode of onset of symptoms, duration of symptoms and previous treatment history were sought from the participants

Analysis of plasma level of serotonin

Plasma serotonin concentration was measured by using enzyme linked immune assay (ELISA) technique. The serotonin assay kit used in this study manufactured by Labor Diagnostika, Germany. The sensivity of the kit is $5 \mathrm{ng} / \mathrm{ml}$ with less than $5 \%$ intra assay variation and $6 \%$ inter assay variation. It has a cross reactivity of $100 \%$ and its linearity in the serum is $100 \%$.

Participants were asked to refrain from smoking cigarette and taking caffeine and/or alcohol at least 12 hours before the exercise testing because this may potentially influenced plasma level of serotonin. In order to minimize the biochemical effects of the previous participants physical activity levels of serotonin, participants were requested to observe an initial 30 minutes rest in supine lying position with pillow support under their head and knees ${ }^{6}$. Collection of blood samples and assaying were carried out between 9am and $11 \mathrm{am}$ in order to control for any possible diurnal variation effects on plasma levels of serotonin ${ }^{6}$.

Blood samples $(10 \mathrm{ml})$ were collected from the antecubital vein into the syringes after 30 minutes of bed rest in a supine lying and then transferred into a tube containing ethylenediaminetriacetic acid (EDTA). The EDTA tube was gently rocked several times to prevent coagulation before beign transferred to a centrifuge. The blood was then centrifuged at $1600 \mathrm{xg}$ for 15 minutes. Extraction of peptides and assaying for the level of plasma serotonin was carried out according to the instruction of the manufacturer and in a similar way as described by Sokunbi et $\mathrm{al}^{3,5}$. After the initial blood taking participants in the exercise groups were asked to rest for another 5 minutes to observe for any changes in the cardiovascular parameters prior to commencing any further physical activities.

Exercise treatment group

\section{Interventions}

The participants in the exercise group underwent exercise group underwent stabilization exercise treatment for a period of six weeks at a frequency ranging from one to three times a week depending on the 
group allocation. A model of stabilization exercise programme presented in this study began with audio visual information related to the spine and how the spine and local stability muscles work together to protect the spine was provided to aid mental imagery of correct performance prior to carrying out proper stabilization exercise protocol. Stabilization exercise protocols used in this study were similar to the one used in the previously reported studies ${ }^{2,3,5}$

Control Group

Participants allocated to the control group received no exercise treatment during the time the study was carried out.

\section{Post intervention assessments}

Assessment of the plasma levels of serotonin was carried out as previously described

I) Before and immediately after stabilization exercises in all the exercise groups

II) Before and after a period of 30 minutes of resting in a supine position for all the participants in the control group

\section{Statistical analysis}

Data on age, height weight and body mass index (BMI) were presented as mean and standard deviation while comparison between groups were carried out using independent t-test. Data on plasma level of serotonin were tested in an exploratory fashion for normality of distribution, plasma level of serotonin did not show symmetry distribution, thus they were presented as Median and semi interquartile range and a non parametric , Kruskal wallis test was used to analyze' between group' differences in plasma levels of serotonin while post hoc analysis was carried out using Wilcoxon test. Statistical significance was set at $\mathrm{P} \leq 0.05$

\section{Results}

Figure 1 shows the methodological flow chart illustrating the procedure from recruitment of the subject to post intervention assessment

Data on participants' demographic characteristics are presented in table 1, Age, Weight and Height and BMI did not differ significantly among the groups $(\mathrm{P}>0.05)$

The median and the semi interquartile range scores of participants' plasma level of serotonin at baseline, 6 week and 18 weeks assessment are presented in table 2 . Kruskal wallis test showed significant difference in the plasma levels of serotonin across the group at 6 week $(\mathrm{P}=0.01)$ and 18 week $(\mathrm{P}=0.03)$ assessments. Surprisingly, the once weekly ( 1 wkly) and twice weekly ( 2 wkly) groups recorded a decline in the plasma level of serotonin at 18 week follow up while the three times weekly (3wkly) maintained the increased in plasma level of serotonin till 18 week follow up assessment.

Table 3 showed post hoc analysis of the difference in plasma serotonin levels at 6 week. Plasma levels of serotonin were significantly higher in all the exercise groups than the control group. Plasma level of serotonin did not differ significantly between the 1 wkly and 2 wkly group after 6 week of treatment $(P=0.056)$. Plasma level of serotonin in the 3 wkly group was significantly higher than the rest of the exercise groups

Table 4 showed the post hoc analysis of plasma level of serotonin at 18 week follow up assessment. Three times weekly (3wkly) group showed statistically significant higher levels of plasma serotonin in comparison with other groups at 18 week assessment $(\mathrm{P}<0.05)$

\section{Discussion}

The result of this study is consistent with those of previous studies in terms of an increase in the plasma level of serotonin in response to spinal stabilization exercise training ${ }^{3,5}$. Sokunbi et al ${ }^{3,5}$ in 2 different preliminary studies. In one of the studies the response of plasma levels of serotonin to a number of different type of spinal based exercises was investigated in a limited sample size $(n=20)$ of apparently healthy volunteers, thus it might be difficult to extrapolate the findings from this study to population of patient with chronic low back pain disorders. Although, the second preliminary study investigated the effect of stabilization exercises on plasma level in chronic low back pain sufferers, the findings from the study were merely used for the power size calculation and to estimate the minimum sample size that will be required for the present RCT. Moreso, assessments of plasma levels of serotonin were carried out at pre and post exercise levels, without any follow up assessments in either of the preliminary studies. Follow up assessments are useful to provide an insight into long term benefits of stabilization exercise intervention. In addition, the results of previous studies by Sokunbi et $\mathrm{al}^{3,5}$ also bagged two questions: 1) what is an appropriate dosage of treatment that can be expected to achieve a desired outcome in terms of plasma serotonin levels?, 2) what if, any correlation between increase in plasma levels of serotonin and pain intensity levels and functional disability scores in low back pain sufferers? 
The result of the present study has shown that stabilization exercises carried out one weekly, twice weekly and three times a week produced significant increase in the plasma level of serotonin in comparison with the control (no exercise) group after 6 week of treatment. However the three times a week group recorded significant increase in the level of plasma serotonin at 18 week follow up assessments which was not observed in the other two exercise groups. It could be possible that stabilization exercises carried out at a frequency of three times a week or more might achieve optimal and long term biochemical effects in terms of an increase in plasma serotonin levels in chronic low back pain sufferers

It is generally accepted that serotonin is involved in the mechanism of action of exercise induced analgesia, ${ }^{4,7,8}$.. Biosynthesis of serotonin in human body involves hydroxylation of tryptophan (essential amino acid) by tryptophan hydroxylase to produce 5 hydroxyl trytophan (5-HTP) and decarboxylation of 5-HTP by aromatic-L-aminoacid decarboxylase to produce serotonin ${ }^{4}$. It has been established that there are two pools of tryptophan in the blood tissue, One that is bound to the platelets and the other that is free ${ }^{9}$, thus serotonin synthesis is dependent on freee tryptophan availability in the blood tissue. Consequently processes that influence equilibrium between free tryptophan and platelet bound tryptophan will influence serotonin synthesis rate ${ }^{9}$. The efflux of serotonin from platelets is influenced by many factors including raised levels of adrenaline as occur during exercise and other stressful situations ${ }^{9}$.

The increase in plasma serotonin in turn could translate to grater pain reduction and reduction in functional disability caused by low back disorders. However, further studies will be needed to establish if any correlation exist between exercises induced increase in plasma level of serotonin and the levels of pain relief and improvement in functions and quality of life in chronic low back pain sufferers.

Limitation

Factors that could account to the limitation of the present studies might include the relatively higher drop rate in the control group in comparison to the exercise groups. This could be partly due to the fact no treatment was administered in this group. Such a relatively higher dropout rate could be prevented in future study by making a commitment to carry out stabilization exercise treatment to the control group after the completion of the randomized control trial. Participants were also requested to refrain from smoking and taking caffeine and/or alcohol at least 12 hours before pre and post intervention assessments as these could influence plasma concentration of serotonin. However, the authors of this present study could not guarantee total compliance of participants in this regards although question were asked to ensure total compliance prior to initial assessment and exercise testing.

\section{References}

[1] Kidd, BL., Richardson, PM., 'How does neurophysiology affect the signs and symptoms of spinal disorders"? Best Practice Research Clinical Rheumatology 2002, 16:1

[2] Goldby L., Moore, AP., Doust, J., Trew, M., "A randomized controlled clinical trial investigating the efficacy of musculoskeletal therapy on chronic low back disorders" Spine 2006. 31 (10)1083-1093

[3] Sokunbi O., Moore A., Watt, P., 'Plasma levels of beta endorphin and serotonin in response to specific spinal based exercises". South African Journal of physiotherapy $2008,64: 131-37$

[4] Kema IP., deVries EG., Musket FA., 'Clinical Chemistry of serotonin and metabolites'. Journal of Chromatography 2000; $747(1$ 2) $33-48$

[5] Sokunbi O., Watt, P., Moore A. "Changes in plasma level of serotonin in response to spinal stabilization exercises in chronic low back pain patients”. Nig. Qt. J., Hosp. Med 2007,17(3):108-111

[6] Lechin F, Pardey-Maldonado B., VanderDjis B., Orozco B., Lechin AE, Psychoneuroendrochrinology 2004, 29: 669-685

[7] Bulbulian R, 'Endogeneous Opioid effects on Motor neuron pool excitability;Potential analgesic effects of acute exercises". Journal of Manipulative and Physiological Therapeutics 2002; 209-215

[8] Kuraishi Y, Harada Y, Aratani S, Satoh M, Takagi H, Separate involvement of the spinal non adrenergic and serotonergic systems in morphine analgesia. The difference in Mechanical and Thermal analgesic tests. Brain Research 1983;273:245-252

[9] Jensen PN, Mooler HJ and Smith DF. Acute effects of exercise on human blood platelets serotonin uptake and Amino oxidase activity. Biological Psychiatry 1995;38: 125-127 


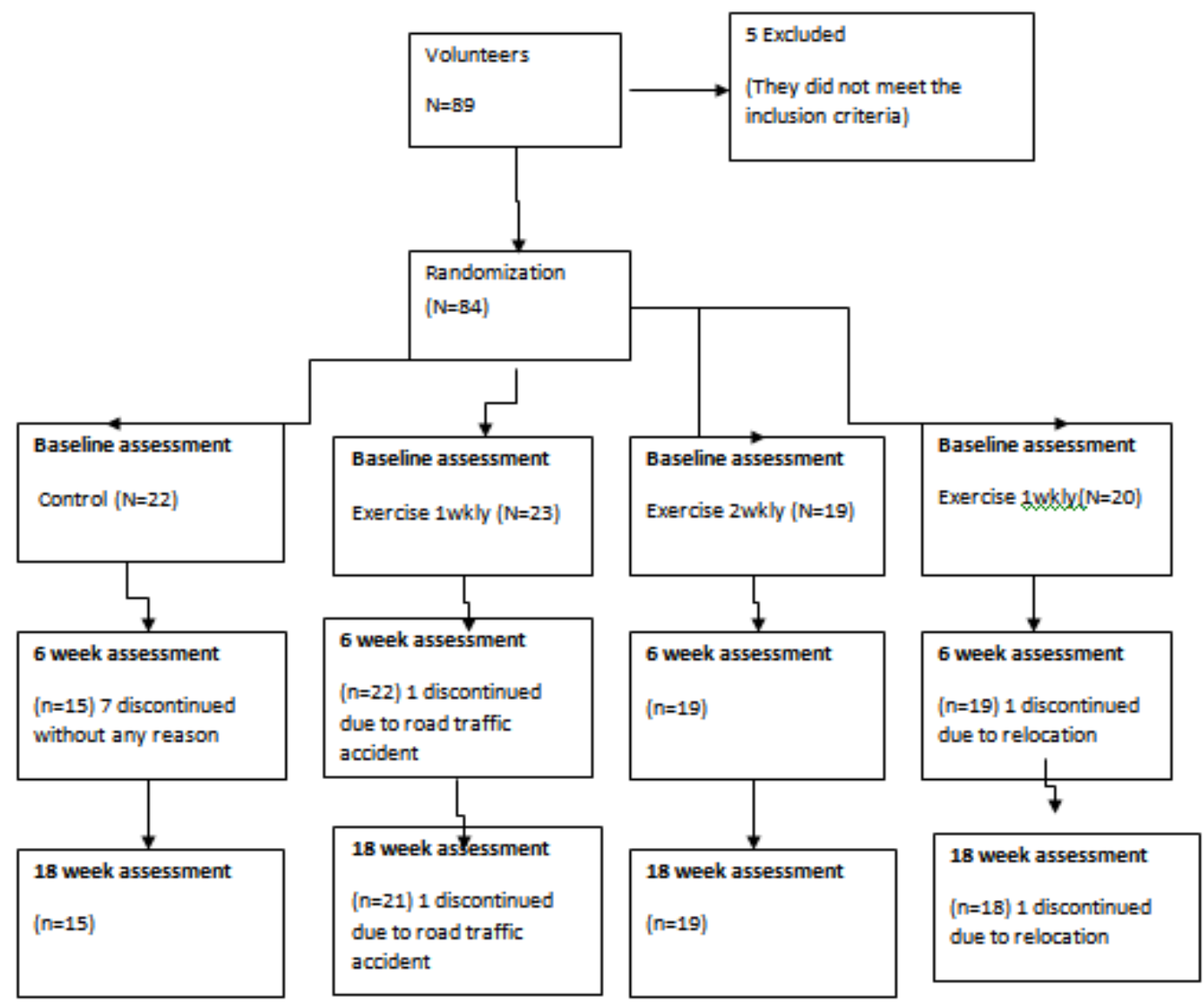

Figure 1 Methodological flow chart of subject recruitments and intervention

Table 2: Participants plasma levels of serotonin at baseline, 6 week and 18 week follow up

\begin{tabular}{|l|l|l|l|l|l|}
\hline & $\begin{array}{l}\text { Control } \\
\text { Median (IQR) }\end{array}$ & $\begin{array}{l}\text { 1wkly } \\
\text { Median (IQR) }\end{array}$ & $\begin{array}{l}\text { 2wkly } \\
\text { Median (IQR) }\end{array}$ & $\begin{array}{l}\text { 3kly } \\
\text { Median (IQR) }\end{array}$ & Pvalues \\
\hline Baseline & $65.0(5.2)$ & $67.4(3.4)$ & $66.8(11.2)$ & $66.8(2.2)$ & 0.333 \\
\hline 6week & $64.8(1.4)$ & $75.2(5.7)$ & $77.8(3.8)$ & $80.0(11.2)$ & $0.01^{*}$ \\
\hline 18 week & $63.0(5.7)$ & $64.0(10.0)$ & $66.9(12.1)$ & $82 . .^{(5.3)}$ & $0.03^{*}$ \\
\hline
\end{tabular}

$*=$ significant

Table 3: Post hoc analysis of Participants plasma levels of serotonin at 6 weeks assessment

\begin{tabular}{|l|l|l|l|l|}
\hline & $\begin{array}{l}\text { Control } \\
\text { P-values }\end{array}$ & $\begin{array}{l}1 \text { wkly } \\
\text { P-values }\end{array}$ & $\begin{array}{l}\text { 2wkly } \\
\text { P-values }\end{array}$ & $\begin{array}{l}\text { 3wkly } \\
\text { P-values }\end{array}$ \\
\hline Control & - & $0.002^{*}$ & $0.007^{*}$ & $0.001^{*}$ \\
\hline 1wkly & $0.002^{*}$ & - & 0.056 & $0.003^{*}$ \\
\hline 2 wkly & $0.007^{*}$ & 0.056 & - & $0.004^{*}$ \\
\hline 3 Wkly & $0.001^{*}$ & $0.03^{*}$ & $0.004^{*}$ & - \\
\hline
\end{tabular}

$*=$ significant

Table 4 : post hoc analysis of Participants plasma levels of serotonin after 18 weeks of treatment

\begin{tabular}{|l|l|l|l|l|}
\hline & $\begin{array}{l}\text { Control } \\
\text { P-values }\end{array}$ & $\begin{array}{l}1 \text { wkly } \\
\text { P-values }\end{array}$ & $\begin{array}{l}\text { 2wkly } \\
\text { P-values }\end{array}$ & $\begin{array}{l}3 \text { wkly } \\
\text { P-values }\end{array}$ \\
\hline Control & - & 0.057 & 0.051 & $0.001^{*}$ \\
\hline 1 wkly & 0.057 & - & 0.06 & $0.003^{*}$ \\
\hline 2 wkly & 0.051 & 0.06 & - & $0.004^{*}$ \\
\hline 3 Wkly & $0.001^{*}$ & $0.003^{*}$ & $0.004^{*}$ & - \\
\hline
\end{tabular}

$*=$ significant 